\title{
EFFECTS OF VERTICAL MISALIGNMENT OF SUPERCONDUCTING COILS IN CYCLOTRONS
}

\author{
Jong-Won Kim* \\ Cyclotron Lab., Michigan State University, E. Lansing, MI 48824, USA
}

\begin{abstract}
A vertical beam loss on the extraction elements of the K500 cyclotron has been observed during commissioning of the Coupled Cyclotron Project. The cause was found to be the misalignment of coils, and the coil shift vertically about $1 \mathrm{~mm}$ made a correction. The vertical displacement of a $9.5 \mathrm{MeV} / \mathrm{u} \mathrm{Ar}$ beam prior to the correction was roughly $4 \mathrm{~mm}$ at a magnetic focusing bar. The effect of coil misalignment seems to be pronounced during beam extraction because the gradient of coil field is high in the extraction region and the magnetic elements employed for radial focusing are misaligned with the coil field. The observed off-center of the Ar beam could also be partly explained by a coherent beam oscillation prior to the extraction if assuming the coil was tilted as the vertical focusing tune approaches one. Balancing of the electromagnetic forces on the coil support links is a usual method of alignment for superconducting coils. In reality a fine adjustment of the coil position may be needed with a beam because readings of the forces on the links could be imprecise indication of the coil position as the coil is supported by the matrix of support links. The coil alignment issue is discussed also for a superconducting separated-sector cyclotron.
\end{abstract}

\section{INTRODUCTION}

The vertical misalignment of coil fields with respect to iron fields in cyclotrons can lead to significant beam losses in the course of beam extraction. During commissioning of the Coupled Cyclotron Project at the NSCL unusually high beam losses have been observed on an extraction magnetic element of the K500 cyclotron. The beam loss was first observed on the beam current profiles measured by the main probe as shown in Fig. 1, where the profile covers the radius ranging from 3 to 31 inches. The rightmost curve in the figure is the current measured at a Faraday cup outside of the cyclotron as the extraction radius of the cyclotron is around 27". The adjacent curve, which has a flat top around 29", is the current measured by the main probe between the fourth magnetic focusing bar M4 and the fifth M5 during the beam extraction. The difference of the two curves corresponds to the beam loss. The beam spot at a diagnostic port just outside of the cyclotron was vertically off by about $5 \mathrm{~mm}$.
The magnetic elements, which are electrically isolated, can be used to measure the beam current, and revealed that the loss was predominantly at the M5. Radiography was taken on the element after irradiation, and showed a vertical shift of the beam center by $4 \mathrm{~mm}$.

Two plausible causes of the beam shift are; 1) superconducting coils displaced from the center of the yoke and pole irons, and 2) extraction elements not vertically centered due to misalignment or due to nonsymmetrical magnetic properties of the steel across the midplane.

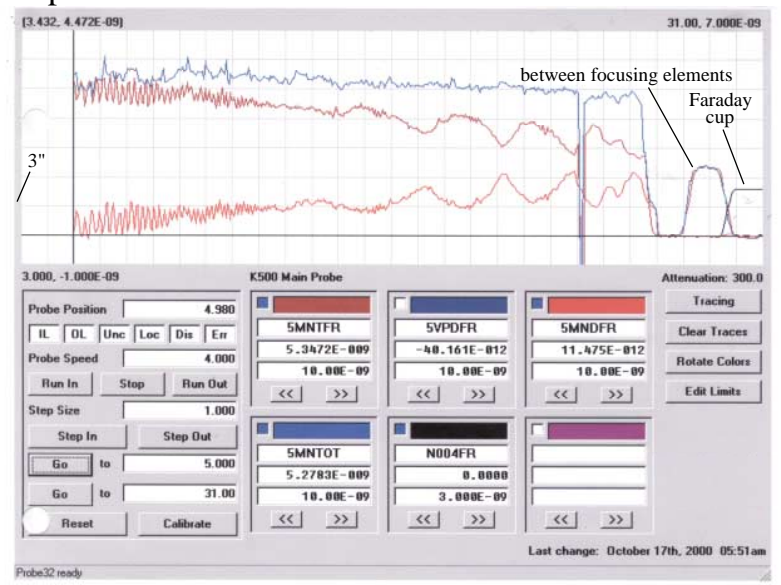

Figure 1: Beam current profiles measured by the main probe of the K500 cyclotron as a function of radius from 3 " to 31 ".

\section{EFFECTS OF THE COIL MISALIGNMENT}

A vertical shift of the coil package by $1 \mathrm{~mm}$ corrected the beam loss problem. The shift was made equally on all six vertical support links by adjusting the bolts. But it is also feasible that the shift could have been unequal due to the presence of rigid attachment to the coil vessel connected from cryostat such as cryolines.

We have performed numerical simulations on the vertical motion of a beam under extraction when the coil is displaced or tilted from the center of iron fields, using the NSCL extraction orbit program that handles the magnetic field linearly in $\mathrm{z}$. The program was modified to add a new term containing the $\mathrm{B}_{\mathrm{r}}(\mathrm{r})$ in the equations of motion to simulate the effects of the coil off-center. The change in $\mathrm{B}_{\mathrm{Z}}(\mathrm{r})$ on the midplane is second order, and thus

\footnotetext{
* This work is supported by the NSF grant No. PHY-9528844
} 
was not considered in this study. The radial profile of the $\mathrm{B}_{\mathrm{r}}(\mathrm{r})$ is determined by the radial gradient of the coil field as shown in Fig. 2. Figure 3 shows the radius of a vertically centered Ar beam in the course of extraction, its $\mathrm{z} \& \mathrm{P}_{\mathrm{z}}$, and beam envelopes as a function of azimuth for two cases: 1) when the coil shift is $1 \mathrm{~mm}$, and 2) when the coil tilt is $1 \mathrm{~mm}$ at the radius of $84 \mathrm{~cm}$. In the case 2) phase of the first harmonic of the $\mathrm{B}_{\mathrm{r}}(\mathrm{r})$ was adjusted to produce a maximum $\Delta z$ at the M5. It appears that the observed $4 \mathrm{~mm}$ off-center at the M5 can be partly explained either by $1 \mathrm{~mm}$ of coil shift or by $1 \mathrm{~mm}$ coil tilt.

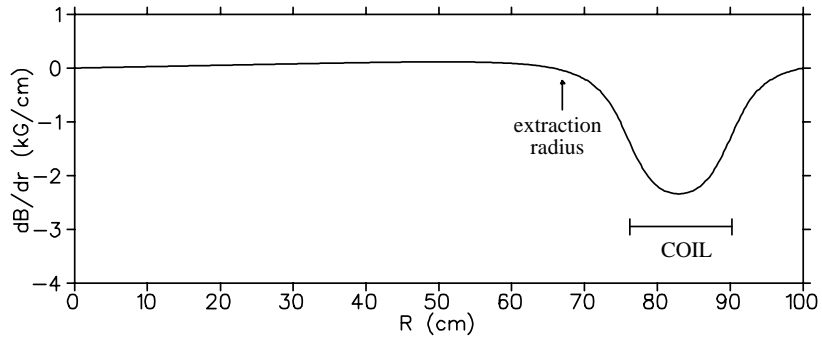

Figure 2: Radial gradient of the $B_{z}$ field solely produced by the K500 coils for the Ar beam.
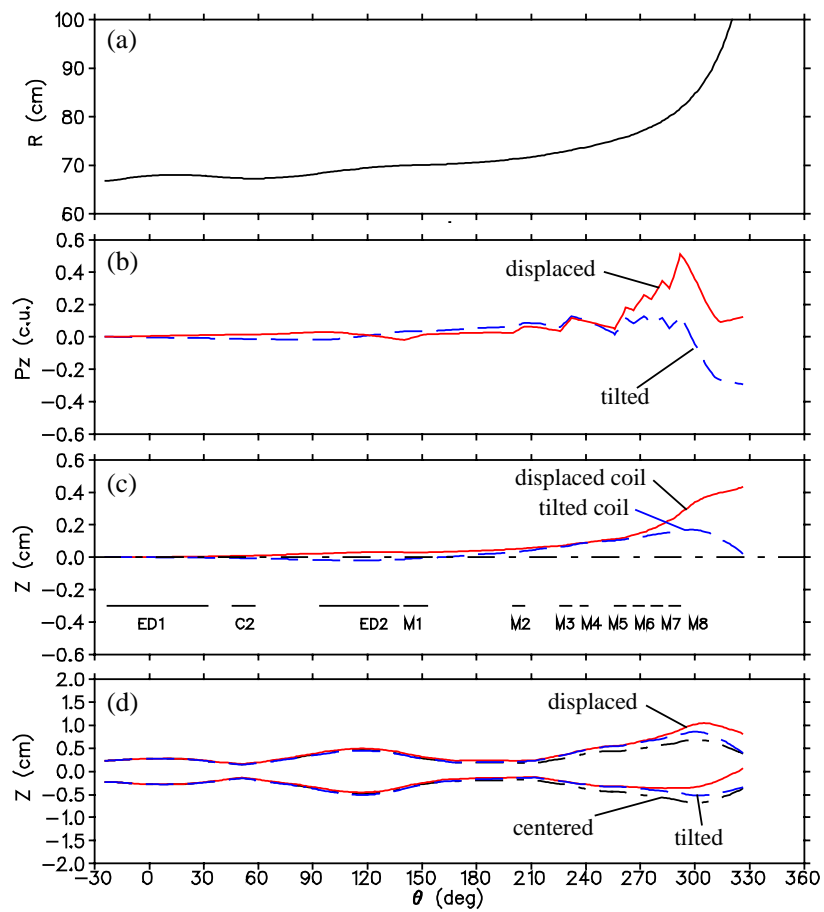

Figure 3: (a) The radius of an extracted $\mathrm{Ar}$ beam as a function of azimuth. (b) \& (c) Motions of $\mathrm{z} \& \mathrm{P}_{\mathrm{z}}$ of the beam that starts at the midplane of iron fields in the presence of the $B_{r}$, (d) Beam envelopes when the coil is centered, displaced, and tilted.

The coil shift could have been unequal because rigid attachment to the coil vessel such as cryolines can affect the force balance. If the coil was tilted, the horizontal first harmonic $B_{r}$ induces a coherent oscillation of a beam prior to the extraction as the vertical tune approaches one as shown in Fig. 4. Near the integral resonance the oscillation amplitude is given as below:

$$
z_{e o}(\omega t)=r \frac{<B_{r}>}{<B_{z}>} \frac{1}{v_{z}^{2}-1} \cos (\omega t+\phi),
$$

where the bracket means an azimuthal average. If assuming the coil was tilted by $0.5 \mathrm{~mm}$ at the links, the $\left\langle\mathrm{B}_{\mathrm{r}}\right\rangle$ is about 10 gauss. Then the oscillation amplitude is approximately $1 \mathrm{~mm}$ at the $v_{z}$ of 0.9 . This could also partly explain the beam off-center. The magnitude of this beam oscillation is rather small for the detection with internal beam probes. The internal beam profiles viewed on a movable scintillator with a borescope in fact showed that the beam was relatively well centered vertically before entering the extraction channels.

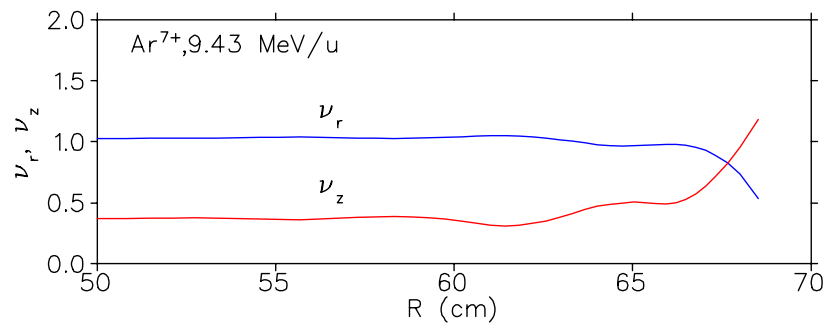

Figure 4: Focusing tunes of the $9.4 \mathrm{MeV} / \mathrm{u}$ Ar beam near the extraction.

\section{MIDPLANE ERRORS BY EXTRACTION ELEMENTS}

A large localized disturbance on the midplane symmetry due to unsymmetrical magnetic elements can cause a vertical shift of a beam. To estimate the magnitude of such fields, an iron cylinder $(1 \mathrm{~cm}$ OD $\times 1$ $\mathrm{cm}$ in $\mathrm{z}$ ) located in one side of the midplane was used as shown in Fig. 5. The field was calculated using a saturated iron approximation, which should be valid in the high field region of extraction. As shown in the inset of Fig. 5, the $B_{r}$ is highly nonlinear, so that a full 3-d field is actually needed to accurately simulate the beam motion. An approximate evaluation was performed considering only the first harmonic component of the iron field. The zdependence of the $B_{r}$ field was parameterised in the equations of motion. The amplitude of the first harmonic $\mathrm{B}_{\mathrm{r}}$ is roughly 5 gauss in the midplane for the assumed iron configuration. The resulting effect on the beam oscillation is less significant than by the coil tilt of $0.5 \mathrm{~mm}$ as the vertical beam envelope is well below the vertical placement of the iron as can be seen in Fig. 3. A major influence may be on the internal beam because multiple turns are affected compared to the single turn during extraction. A consistent orbit calculation including both acceleration and extraction will take much more efforts.

The magnetic extraction channels are placed in a narrow space with a vertical gap of around $3 \mathrm{~cm}$, and thus the misalignment within the gap should be negligible. A larger error can come from the misalignment of cryostat with respect to the coil. 


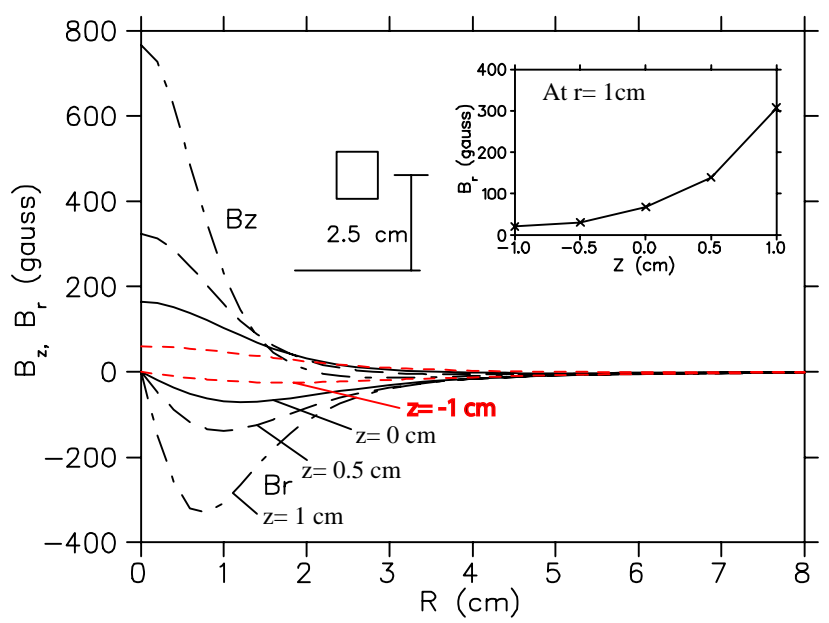

Figure 5: Magnetic fields by an iron cylinder off the cyclotron midplane. The radial origin of abscissa is the center of the iron. The inset is the plot of the $B_{r}$ as a function of $\mathrm{z}$ at the radius of $1 \mathrm{~cm}$.

\section{ALIGNMENT OF COIL}

Minimizing the electromagnetic off-centering forces on thermally insulating support links is a usual method of the coil alignment for the NSCL superconducting cyclotrons[1]. The links are anchored onto the yoke steel thru $2.54 \mathrm{~cm}$ OD strain gages that can support up to 50 tons each. The spring constant at the operating current of $500 \mathrm{~A}$ is around $10^{6} \mathrm{~N} / \mathrm{m}$. Thus a $1 \mathrm{~mm}$ displacement produces $1000 \mathrm{~N}$ of off-centering force, which allows sensitivity needed in coil centering.

If the coil is kept at $4 \mathrm{~K}$, the centering is not expected to change. But the magnet needs to warm up for maintenance or due to failures in cryogenic/vacuum systems. Then there is a chance for reoccurrence of the centering errors. Since the beam test is arguably the most accurate measure for misalignment, we can redo the centering with an easy-to-run test beam, but it still takes a considerable effort. A new method has been explored for the AGOR superconducting cyclotron magnet to find out the midplanes of the coil and pole iron fields[2]. Two Hall probes placed equally across the mdiplane are used while error signals are nullified by another measurement with the probe set $180^{\circ}$ rotated. Other attempt has been made for a separated-sector cyclotron[3].

\section{ALIGNMENT ISSUE IN A SEPARATED- SECTOR CYCLOTRON}

The design of superconducting separated-sector cyclotrons for high-energy heavy ion acceleration such as the SRC under construction at RIKEN usually asks for detailed assessments on alignment tolerances for various cyclotron elements. Some early discussions on the SRC have been made as in ref. [4], and more detailed evaluations in ref. [5] for the final magnet configuration. The analysis in fact has been made largely based on criticality of integer and half integer resonances as different ions pass thru or approaches to different resonances during acceleration. On the other hand effects on the orbit during injection and extraction by the coil misalignment seem to be further investigated. The modes of coil misalignment are more complicated for the sector coils than for the circular coils. Hence, simulations of vertical beam off-centering for some representative modes could be useful to find out the corrective adjustment of coil positions if a beam is found to be vertically offcentered.

\section{CONCLUSION}

The vertical beam loss on the extraction elements has been corrected with a vertical coil shift of $1 \mathrm{~mm}$. Once the coil was adjusted for a better alignment, the beam loss during the extraction became negligible with a careful tuning on the extraction elements. The mismatch in magnetic centers between the coil fields and ithe ron fields seems to sensitively appear as the vertical off-center of a beam in the extraction region. The coil shift could be reoccurring when the magnet temperature recycles between $4 \mathrm{~K}$ and $293 \mathrm{~K}$, but it may be easily correctable owing to accumulated experiences on the coil alignment.

\section{REFERENCES}

[1] P. Miller, et al., NSCL Annual Report (1978) p117.

[2] H. Schreuder, a note from private communication, (2000).

[3] L. Schulein and H. Jungwirth, NIM A 401, (1997) p171.

[4] J. Kim et al., Rev. of Sci. Instr. V70, No.5, (1999) p2293.

[5] A. Goto, Internal note, Estimations on tolerance for construction and assembly of the SRC sector magnet, RIKEN (2001). 